\title{
Computergestützte Osteotomieplanung
}

\author{
Christian Dahlen, Michael Amlang, Hans Zwipp
}

\section{Zusammenfassung}

Korrekturosteotomien sind elektive Eingriffe, die einer sorgfältigen und vollständigen präoperativen Planung bedürfen, um das operative Ziel sicher und ohne Komplikationen zu erreichen. In komplexen räumlichen Konstellationen mit multiplanaren Fehlstellungen ist die konventionelle Röntgendiagnostik einschließlich der Computertomographie oftmals für die Erfassung der dreidimensionalen Problematik nicht ausreichend. In Einzelfällen wurde deshalb für die Operationsplanung mit erheblichem, zeitlichem und finanziellen Aufwand ein reales Modell auf Grundlage einer Computertomographie angefertigt. Der rasante Anstieg der Rechenleistung in der EDV ermöglicht inzwischen eine schnelle 3D-Modellerstel-

\section{Einleitung}

Osteotomien sind ein häufiges Operationsverfahren in der Chirurgie des knöchernen Bewegungsapparates, um angeborene, erworbene oder posttraumatische Fehlstellungen zu korrigieren. Sie finden an allen Lokalisationen des Skeletts Anwendung, vom Schädel hinab bis zu den Zehen. An den langen Röhrenknochen der Extremitäten werden Achsfehlstellungen, Torsionsfehler und Längendifferenzen korrigiert, wenn sie zu einer Funktionsbehinderung oder zu einer unphysiologischen Belastung der Gelenke führen. Auch in kompakten knöchernen Regionen wie dem Gesichtsschädel [28], der Wirbelsäule, dem Azetabulum $[15,16]$ und dem Fuß [32] werden

OP-JOURNAL 2002; 17: 40-44

(c) Georg Thieme Verlag Stuttgart . New York lung mit dem Computer. Mit einer eigens für diese Anwendung entwickelten 3D-Software, die auf einem PC unter Windows eingesetzt wird, kann an einem virtuellen Modell eine vollständige dreidimensionale Analyse und alle erforderlichen Planungsschritte einer Korrekturosteotomie vorgenommen werden. Gegenüber der Planung an einem realen Modell bietet sie weitaus bessere Analyse-, Simulations- und Vermessungsmöglichkeiten. Die Software ist einfach zu bedienen und aufgrund ihrer Modularität erweiterungsfähig und individuellen Anforderungen und Arbeitsabläufen einfach anzupassen. Sie bietet auch für Forschung- und Lehraufgaben alle erforderlichen Funktionen für eine dreidimensionale Aufgabenbearbeitung.
Für einfache unifokale Fehlstellungen genügen an bildgebender Diagnostik in den meisten Fällen technisch einwandfreie konventionelle Standard-Röntgenaufnahmen, eventuell ergänzt durch eine Computertomographie. Dagegen sind bei mehrdimensionalen Fehlstellungen aufgrund der gegenseitigen dreidimensionalen Abhängigkeit der einzelnen Fehlstellungskomponenten eine exakte Analyse und nachfolgende Planung mit herkömmlichen Methoden äußerst schwierig, wenn nicht unmöglich.

Anspruchsvolle Korrekturosteotomien erfordern eine vollständige, detaillierte und präzis dokumentierte präoperative Planung.

Im Einzelfall wurde daher für die Operationsplanung in diesen komplexen $\mathrm{Si}$ tuationen auf ein reales Modell $[9,25]$ zurückgegriffen, das im Fräsverfahren [20] oder mittels Stereolithographie $[3,14,21]$ in der Regel auf der Grundlage einer Computertomographie angefertigt wurde. An diesen Modellen wurden die Fehlstellung analysiert und die Korrekturoperation geplant bzw. ausprobiert. Die Herstellung dieser Modelle war jedoch zeitaufwändig und teuer. Weitere Nachteile bestanden in einer ungenügenden räumlichen Analyse sowie bei den Stereolithographien in einer schwierigen Bearbeitung des zäh-elastischen Kunststoffpolymers.

chen. Während der Planung wird die Fehlstellung analysiert, das Operationsverfahren und der Zugang ausgewählt, die Art und Lokalisation der Osteotomie festgelegt, eine Vorauswahl des Implantats getroffen und überprüft, ob sich das angestrebte Ergebnis erreichen lässt. Intraoperative Probleme können durch die Planung frühzeitig erkannt und alternative Lösungen gesucht werden. Das postoperative Ergebnis sollte mit der dokumentierten Planung verglichen werden - ein wesentlicher Beitrag zur Qualitätssicherung und Schulung der chirurgischen Qualifikation.
Die Computertomographie erfasst das Untersuchungsvolumen mit hoher Ortsauflösung [10] und ermöglicht die Berechnung realitätsnaher virtueller 3DModelle. Auf dieser Grundlage hat sich in den letzten beiden Jahrzehnten eine computergestützte Chirurgie $[2,4,5,7$, 19] entwickelt, die auch unter dem Begriff CAOS („Computer aided orthopaedic surgery“) bekannt wurde. Neben der dreidimensionalen Visualisierung bietet sie auch Möglichkeiten für das „Rapid Prototyping“ [30] und die intraoperative Navigation [22]. Die virtuellen 3D-Modelle 
wurden bereits von verschiedenen Softwareentwicklungen zur Operationsplanung herangezogen $[6,9,13,31]$, ohne dass bislang ein ausgereiftes, universell einsetzbares Softwareprodukt zur Verfügung stand.

\section{D-Planungsoftware für PC}

Für die virtuelle dreidimensionale Operationsplanung wurde in enger Zusammenarbeit mit der im Bereich der medizinischen Bildverarbeitung spezialisierten Firma IVS (Integrierte Visualisierungssysteme) Software Engineering AG/Chemnitz eine CE-zertifizierte modulare Software entwickelt. Diese wird auf einem handelsüblichen Arbeitsplatz-PC (Abb. 1) unter einem Windows-Betriebssystem eingesetzt und benötigt keine besondere Hardwareausstattung. Zur Bearbeitung umfangreicher Datensätze ist allerdings ein ausreichend dimensionierter Hauptspeicher mit $256 \mathrm{MB}$, besser jedoch mit $512 \mathrm{MB}$ erforderlich, um einen zügigen Arbeitsfluss zu gewährleisten und rechenintensive 3D-Rekonstruktionen in nahezu Echtzeit [26] darzustellen.

Über eine DICOM-III-Schnittstelle können nicht nur CT- und MRT-Schnittbilder, sondern jede beliebige Schnittbilduntersuchung eingelesen werden.

Nach einer strengen Konsistenzprüfung wird aus den Schnittbildern ein systeminterner Volumendatensatz generiert, der die gesamte Information des Untersuchungsvolumens repräsentiert. Bei einer Schnittbildgröße von $512 \mathrm{~KB}$ ist dieser in der Regel 50-70 MB groß - er bildet die Basis für alle Funktionen 3D-Software VoXim (Voxel Imaging). Infolge des voxelbasierten Darstellungsprinzips [11,17,18, 23,29] bleibt innerhalb der 3D-Objekte jederzeit die gesamte Volumeninformation erhalten.

\section{D-Visualisierung}

Der Volumendatensatz kann neben der primären transversalen Schnittebene in der rekonstruierten sagittalen und koronaren Standardebene als auch in beliebigen schrägen Anschnitten betrachtet werden (Abb.2au.b). Neben einer Einzelbildbetrachtung ermöglicht ein dynamisches Scannen des Datensatzes bereits einen guten Überblick über räumliche Beziehungen anatomischer Strukturen. Spezielle 2D-Rekonstruktionen an gekrümmten Flächen sind in der Software implementiert. Sie eignen sich, um z.B. die gewölbte Lisfranc-Gelenkreihe auf

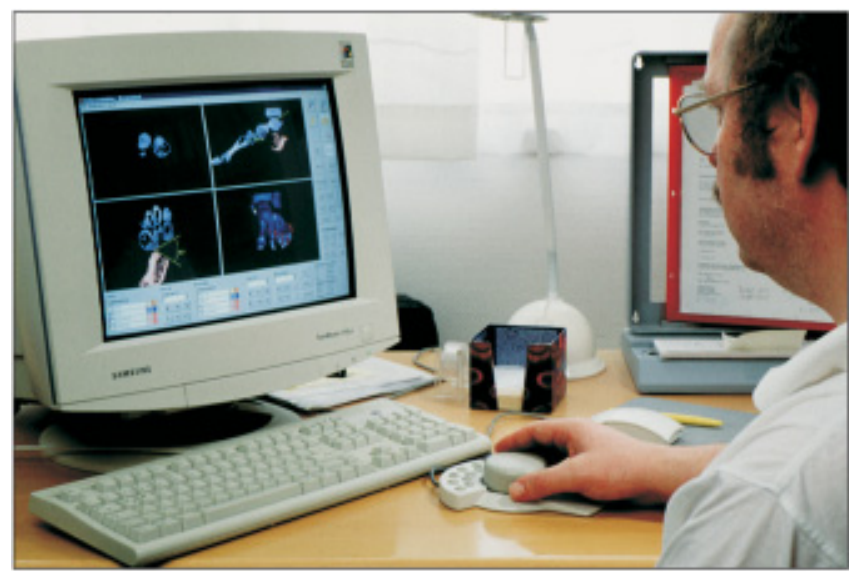

Abb. 1 3D-Operationsplanung am $\mathrm{Ar}$ beitsplatz-PC. Mit einer 3D-Maus wird die Osteotomiesimulation einer Rückfußkorrektur gesteuert.

eine Ebene zu projizieren, finden aber wegen ihrer Verzerrungen in der linearen Operationsplanung keine Verwendung. Zusätzliche Funktionen wie Ausschnittsvergrößerungen, Kontrast- und Helligkeitsregelung sowie geometrische Vermessungswerkzeuge bieten die gleiche Funktionalität wie die Bildbearbeitungssoftware moderner Computer- oder Magnetresonanztomographen.

\section{D-Rekonstruktion}

Eine plastische dreidimensionale Darstellung einzelner anatomischer Objekte wird aber erst durch eine Segmentation des Volumendatensatzes möglich. Mit diesem Verfahren werden die relevanten Objekte aus ihrer Umgebung herausgelöst (Abb.2cu.4).

Knöcherne Strukturen im CT-Volumendatensatz können in den meisten Fällen automatisch mit einem Schwellwertverfahren segmentiert werden, da große Dichteunterschiede zwischen Knochen und Weichteilen eine sichere Zuordnung erlauben.

Dagegen muss die Segmentation im MRT mit verschiedenen grafischen Hilfsmitteln manuell Schicht für Schicht vorgenommen werden, was bei vielen Schichten einen sehr hohen Arbeitsaufwand bedeutet. Zur virtuellen Planung von Korrekturosteotomien werden daher, auch wegen der hervorragenden Ortsauflösung, in der Regel nur CT-Datensätze verwendet. Wenn erforderlich, kann die ursprüngliche Segmentation im „Region growing"-Verfahren in bis zu 8 Subsegmentationen untergliedert werden.

Für die Visualisierung der segmentierten 3D-Objekte sind zahlreiche Funktionen verfügbar. Zur Erfassung seiner dreidi- mensionalen Form lässt sich das Objekt um alle 6 Freiheitsgrade mit zahlreichen, z.T. auch intuitiven Steuerungsfunktionen bewegen. Die Segmentationen können verschiedenfarbig eingefärbt und im Transparenzmodus auch innen- oder dahinterliegende Strukturen betrachtet werden. Mit unterschiedlichen räumlichen Anschnitten ist auch eine direkte Einsicht in die Tiefe der Objekte möglich. Der dreidimensionale Eindruck [27] wird durch eine stereoskopische Betrachtung mit einer LCD-Shutterbrille [1] oder bei Betrachtung auf einem 3D-Monitor (z.B. autostereoskopisches Display der Fa. Dresden 3D GmbH) eindrucksvoll verstärkt. Damit kann ein Nachteil der virtuellen Darstellung, die fehlende Haptik, wenigstens zum Teil ausgeglichen werden.

Verschiedene Messfunktionen gestatten eine komfortable Bestimmung von Strecken, Winkeln, Flächen und Volumina (Abb.3u.4). Geometrische Orientierungshilfen wie Punkte, Linien und Ebenen unterstützen die dreidimensionale Vermessung und Positionierung von Objekten. Die Orientierungshilfen können entweder intuitiv frei im Raum platziert oder an anatomischen Landmarken ausgerichtet werden. Ihre farbige Darstellung und die Möglichkeit, sie nach Bedarf ein- und auszublenden ohne ihre Lage zu verändern, erleichtern die Übersicht in komplexen Situationen.

\section{Anatomisches Koordinatensystem}

Sowohl für die dreidimensionale Analyse als auch für die Manipulationen der Operationssimulation ist die Definition eines eigenständigen orthogonalen Koordinatensystems unverzichtbar, das sich an der Struktur des jeweiligen anatomischen Bereichs orientiert. Für Planungen 

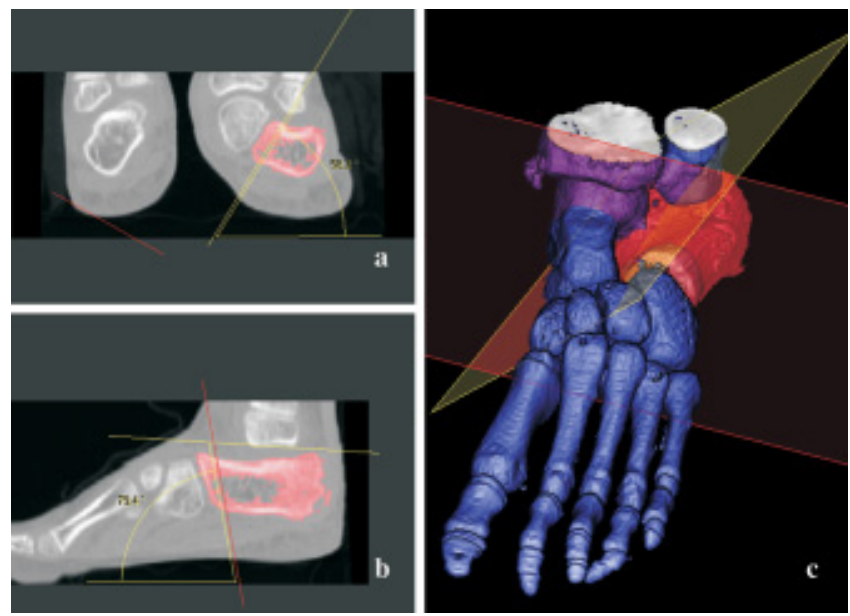

Abb.2a-c Planung einer biplanaren extraartikulären Korrekturosteotomie des linken Kalkaneus bei einem extremen Pes planovalgus eines 8-jährigen Jungen mit einem Dysmelie-Syndrom. (a) Koronare 2D-Rekonstruktion: Die subtalare Osteotomieebene (gelb) weist in Bezug zur Plantarebene einen Winkel von 58,2 $2^{\circ}$ auf. (b) In der sagittalen 2D-Rekonstruktion verläuft die Osteotomieebene durch den Processus anterior calcanei (rot) in einem Winkel von 79,4 zur Plantarebene. (c) 3D-Darstellung des linken Fußes mit eingeblendeten Osteotomieebenen.

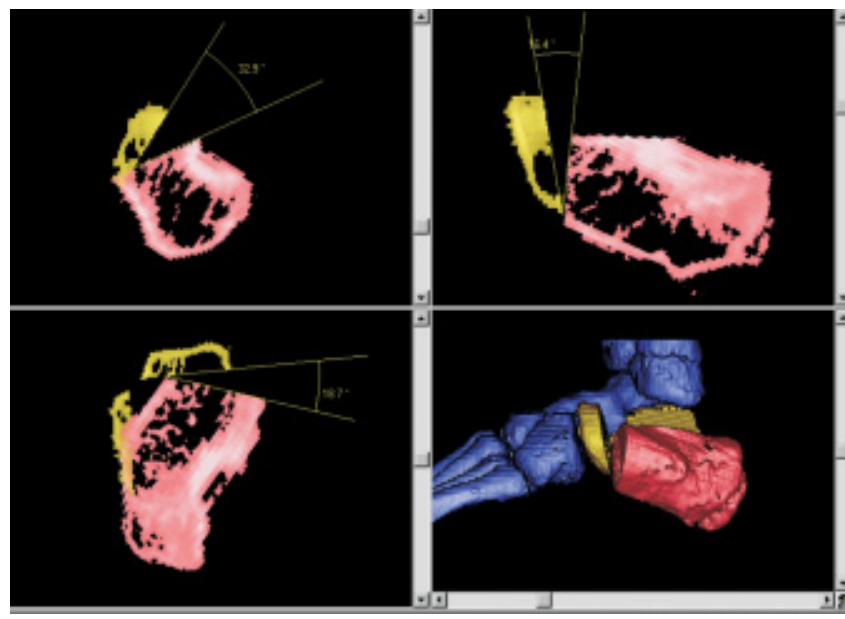

Abb. 3 Nach Ausführung der virtuellen Kalkaneusosteotomien (siehe Abb. 2) erfolgte die Rückfußaufrichtung zur Normalisierung der Fußstatik. Vermessung der Osteotomiewinkel in den Standardebenen. Die seitliche 3D-Ansicht rechts unten zeigt die Position des Kalkaneuskorpus (rot) nach der Reorientierung.
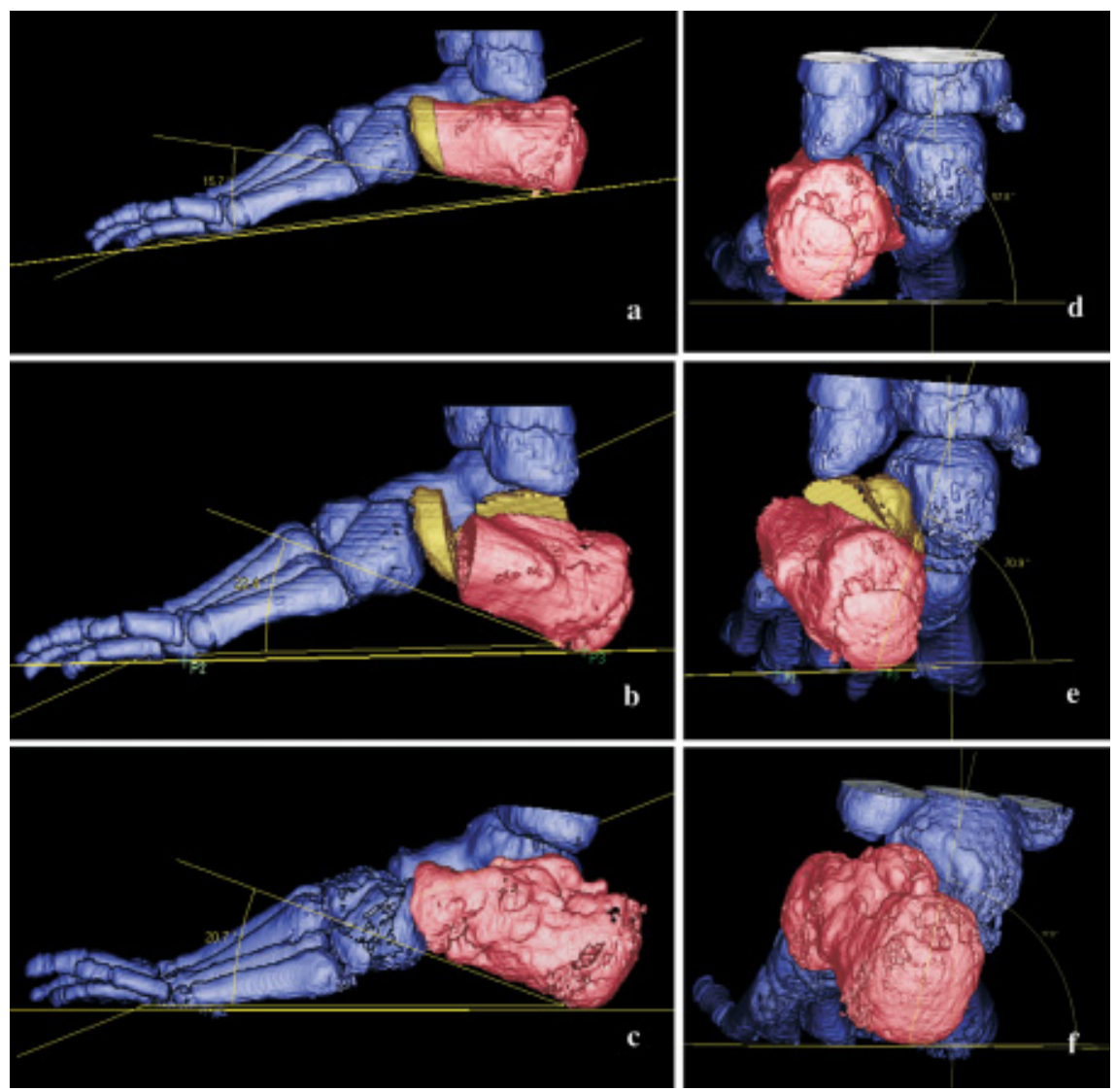

Abb.4a-f Standardisierte Seiten- und Rückfußansicht vom vorherigen Fall aus Abb. 2 und 3, dargestellt in einem anatomischen Koordinatensystem. Die präoperative Situation wird mit der simulierten Korrektur und dem erreichten postoperativen Ergebnis verglichen. Vermessung der Kalkaneusinklination als seitlicher Parameter für die Rückfußstellung. (a) Diese betrug präoperativ $15,7^{\circ}$, (b) nach der virtuellen biplanaren periartikulären Osteotomie $22,4^{\circ}$ und (c) in der postoperativen CT-Kontrolle $20,7^{\circ}$. (d) Präoperativer Winkel der Rückfußachse: 57, ${ }^{\circ}$, (e) nach der Simulation $70,9^{\circ}$ und (f) postoperativ $77,8^{\circ}$. am Fuß z.B. bieten sich die Plantarebene und die Ausrichtung der Talometatarsale I-Achse als geometrische Kriterien für die Einrichtung des neuen Koordinatensystems an. Dieses ermöglicht eine standardisierte Vermessung von dreidimensionalen Beziehungen sowie von projizierten Winkeln und Distanzen (Abb.4). Nicht nur die Standardansichten des 3D-Objekts, sondern auch alle 2D-Rekonstruktionen werden nach dem anatomischen Koordinatensystem ausgerichtet.

\section{Planungsablauf einer Osteotomie}

Erster Schritt einer Planung ist eine umfassende Analyse der knöchernen Pathomorphologie, die sich vergleichend an der gesunden Gegenseite oder an bekannten anatomischen Formen und Gesetzmäßigkeiten orientiert. Dabei weist jede anatomische Region eigene Schwerpunkte und Prinzipien auf. So gilt für den Fuß die Wiederherstellung der normalen Statik als wichtigstes Prinzip [32], dagegen für die Beinachse die Ausrichtung der Gelenkflächen in Bezug zur Tragachse [24].

Mit Unterstützung des anatomischen Koordinatensystems, der geometrischen Orientierungshilfen und von Messwerkzeugen lässt sich in den 2D- und 3D-Rekonstruktionen auch in komplexen Konstellationen eine vollständige dreidimensionale Analyse vornehmen. 

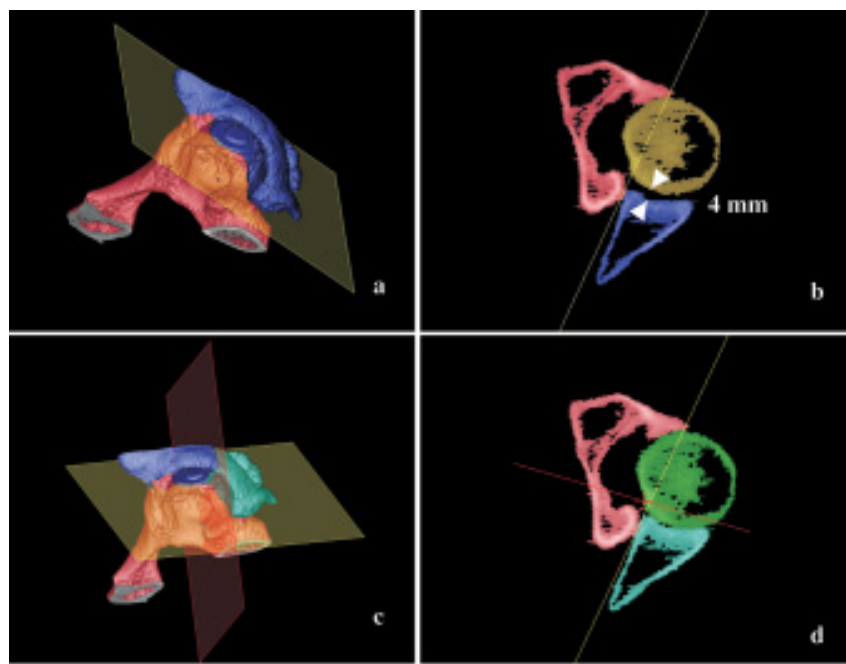

Abb.5a-d Fehlverheilte transtektale Transversalfraktur linkes Azetabulum bei einer 32-jährigen Patientin mit einer Inkongruenz der posterioren Gelenkfläche. (a) 3D-Ansicht von laterokaudal mit ausgeblendetem Hüftkopf. Die gelbe Ebene kennzeichnet die ehemalige Frakturebene. (b) Im Transversalschnitt durch das Hüftkopfzentrum zeigt sich eine Inkongruenz im Bereich des hinteren Pfeilers (blau) mit einem auf $4 \mathrm{~mm}$ verbreitertem Gelenkspalt. (c) Planung einer Korrekturosteotomie der hinteren Pfannenwand mit eingeblendeten Osteotomieebenen. (d) Erreichte Gelenkkongruenz durch Translation der osteotomierten Hinterwand in der Operationssimulation.
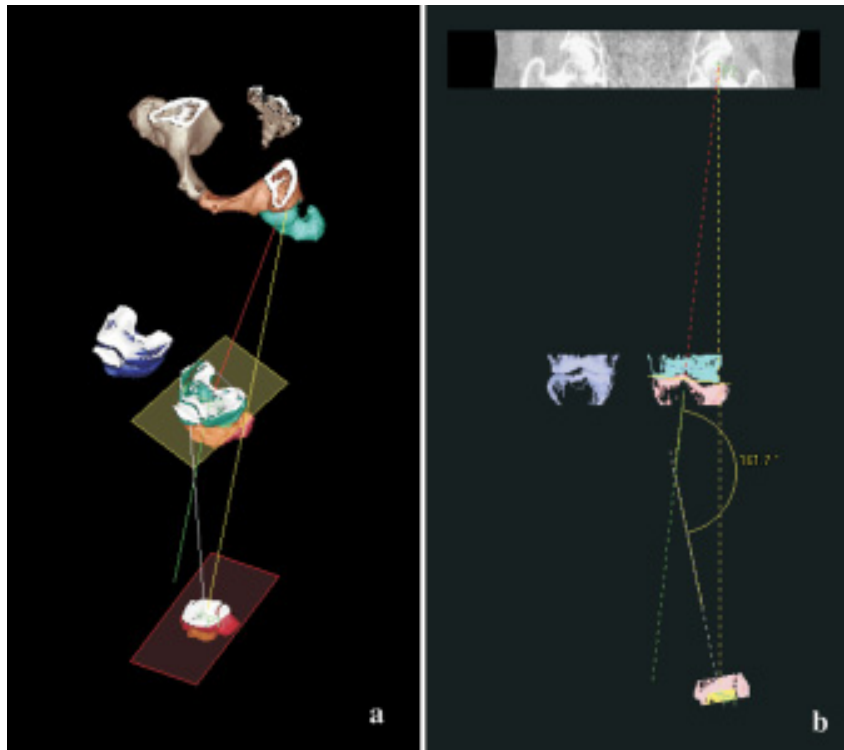

Abb.6au.b (a) 3D-Analyse einer multiplanaren Unterschenkelfehlstellung links. Die Abschnitte des Hüft-, Knie- und Sprunggelenks mit den Normalebenen des Knie- und Sprunggelenks sind eingeblendet. Tragachse - gelbe Linie; mechanische Tibiaschaftachsen - grüne und weiße Linie. (b) In der anatomischen Frontalebene der 2D-Rekonstruktion beträgt die Valguskomponente der kombinierten Valgus-Antekurvations-Außentorsionsfehlstellung 18,3 .
Für die Korrektur der analysierten Fehlstellung sind nach Wahl des Verfahrens die räumliche Lage der Osteotomieebenen, die Positionsänderung von osteotomierten Knochenteilen um die 6 Freiheitsgrade, verbleibende Knochendefekte oder auch kallusbedingter Knochenüberschuss, die Ausführbarkeit der vorgesehenen Operationsmethode als auch das erreichbare Operationsergebnis wichtige Planungsziele (Abb.3-5). Mit den geometrischen Hilfsmitteln werden anatomische Zielkriterien für die Korrekturoperation eingerichtet. Entlang den positionierten Osteotomieebenen (Abb.2cu.5c) wird der Knochen wie mit einem „,virtuellen Osteotom" durchtrennt, was datentechnisch einer Subsegmentierung entspricht. Der osteotomierte Teil wird mit verschiedenen Bewegungsfunktionen an den Leitstrukturen ausgerichtet. Ist das virtuelle Korrekturziel erreicht, wird die Gesamtbewegung des Objekts aus dem Vergleich Ist- zum Sollzustand in seinen Translationen und Rotationen bestimmt. Distanzen und Winkel der Osteotomien, aber auch die Position von anatomischen Landmarken lassen sich präzise messen, als intraoperative Vorgaben verwenden oder mit einem intraoperativen Navigationssystem umsetzen. Einblendbare Implantate im CAD-Format können vorausgewählt und ihre optimale Position vorbestimmt werden.

\section{Korrektur von Extremitätenachsen}

Mehrdimensionale Achsfehlstellungen der Extremitäten sind mittels konventioneller Röntgendiagnostik nur mit kombiniert grafisch-mathematischen Verfahren zu analysieren [12], die schwierig zu handhaben sind und zahlreiche Fehlerquellen aufweisen können. Eine 3D-Analyse ermöglicht auch hier eine genaue Bestimmung der einzelnen Fehlstellungskomponenten. Da die Extremitätenachsen durch die räumliche Position der Gelenkflächen zueinander eindeutig definiert sind, genügt zur 3D-Analyse die Abbildung der einzelnen Gelenkabschnitte im CT (Abb.6). Wird der Bereich der Osteotomie als eigenes 3D-Modell dargestellt, können an dieser Stelle unterschiedliche Osteotomieverfahren simuliert und ihre resultierenden Korrekturwerte in den Standardebenen bestimmt werden.

\section{Weitere Funktionen}

Da die 3D-Software ist ein Hybridsystem mit volumen- und oberflächenbasierten Darstellungsmöglichkeiten ist, verfügt sie noch weitere Funktionen der medizinischen Bildverarbeitung. Beliebige CADObjekte, wie z.B. Implantate, Werkzeuge oder anatomische Strukturen, können eingeblendet, positioniert und sowohl in den 2D- als auch 3D-Rekonstruktionen dargestellt werden. Aus dem Volumendatensatz lassen sich virtuelle Röntgenprojektionen ohne Projektionsfehler rückrechnen, die nach Abschluss der Planung ausgedruckt und als Vorlage für intraoperative Röntgenkontrollen herangezogen werden können. Auch ein Matching von CT- und MRT-Daten ist möglich, um z.B. die Relation von Weichteilstrukturen zum Skelett genau und anschaulich darzustellen.

Verschiedene Exportfunktionen erlauben die Anbindung eines Navigationssystems, die Weiterverwendung der Daten für das Rapid Prototyping oder in anderen CAD-Anwendungen.

\section{Schlussfolgerung}

Die 3D-Planungssoftware VoXim ermöglicht mit aktueller PC-Technik eine umfassende dreidimensionale Analyse und Osteotomiesimulation anhand von CToder auch MRT-Schnittbilduntersuchungen. Der Chirurg kann an seinem Arbeitsplatz zum Zeitpunkt seiner Wahl, unabhängig von den Möglichkeiten in der Radiologie, die Operationsplanung vornehmen. Die hier vorgestellte modulare Software bietet alle dazu erforderlichen Funktionen und ist für individuelle An- 
wendungen uneingeschränkt ausbauund anpassungsfähig.

Durch die eigenständige Beschäftigung mit der Dreidimensionalität komplexer Fehlstellungen wird die Qualität der Operationsvorbereitung gesteigert und das räumliche Verständnis geschärft.

Gerade bei nicht so häufigen Eingriffen mit höherem Schwierigkeitsgrad ist durch die dreidimensionale Operationsvorbereitung ein steilerer Anstieg der Lernkurve zu erwarten. Auch ein Chirurg mit weniger Erfahrung wird sich nach der virtuellen Vorbereitung eher einen anspruchsvollen Eingriff zutrauen.

Für die exakte intraoperative Umsetzung der Osteotomieplanung ist die Anbindung an ein intraoperatives Navigationssystem geplant, mit dem wichtige Operationsschritte überwacht und das real erreichte Ergebnis während der Operation überprüft werden kann.

\section{Literatur}

${ }^{1}$ Bach M. Räumlich durchs Auge. c't 7 1999: 158-162

${ }^{2}$ Berlemann U, Langlotz F, Langlotz U, Nolte LP. Computer assisted orthopaedic surgery. Orthopäde 1997; 26: 463-469

${ }^{3}$ Bill JS, Reuther JF, Dittmann W, Kubler N, Meier JL, Pistner H, Wittenberg G. Stereolithography in oral and maxillofacial operation planning. Int J Oral Maxillofac Surg 1995; 24: 98-103

${ }^{4}$ Blackwell M, Morgan F, Di Gioia AM. Augmented reality and its future in orthopaedics. Clin Orthop 1998; 354: 111-122

${ }^{5}$ Börner M. Computergestützte Chirurgie. Unfallchirurg 1997; 100: 689-691

${ }^{6}$ Burk DL Jr, Mears DC, Cooperstein LA, Herman GT, Udupa JK. Acetabular fractures: three-dimensional computer tomographic imaging and interactive surgical planning. J Comput Tomogr 1986; 10: 1-10

${ }^{7}$ Di Gioia A.M. What is computerassisted orthopaedic surgery? Clinic Orthop 1998; 354: $2-4$

${ }^{8}$ Erhardt J, Handels H, Peters P, Plötz W, Pöppl SJ. Preoperative Planning and Simulation of Pelvic and Hip Endoprosthesis using Virtual Three-dimensional Models. 4th International Symposium of CAOS, Davos 1999: S. 19
${ }^{9}$ Fleiner B, Hoffmeister B, Kreusch T, Lambrecht T. Dreidimensionale Operationsplanung am Modell - eine kritische Bestandsaufnahme. Fortschr-Kiefer-Gesichtschir. 1994; 39: $13-16$

${ }^{10}$ Galanski M, Prokop M. Ganzkörper-Computertomographie. Thieme, Stuttgart New York 1998

${ }^{11}$ Gerig G, Szekely G. Visualisation and Image Processing of Medical Image Data. In: Nolte LP, Ganz R (Eds.): Computer Assisted Orthopedic Surgery, Hogrefe \& Huber, Seattle Toronto Bern Göttingen 1999: S. 1 - 14

${ }^{12}$ Gürke L, Martinoli S. Korrektur mehrdimensionaler Deformitäten durch eine einzige Osteotomie. In: Strecker W, Keppler P, Kinzl L (Hrg): Posttraumatische Beindeformitäten. Springer Berlin Heidelberg 1997: S. $277-$ 283

${ }^{13}$ Hohmann D, Hu S, Koppel R, Legal H, Scheuring, $H$. Approaches in computer-assisted surgical planning in hip dislokation. Orthopäde 1988; 17: $468-471$

${ }^{14}$ Kacl GM, Zanetti M, Amgwerd M, Trentz O, Seifert B, Stucki H, Hodler J. Rapid prototyping (stereolithography) in the management of intra-articular calcaneus fractures. Eur Radiol 1997; 7: 187-191

${ }^{15}$ Klaue K, Bresina S, Guelat P, Wallin A, Perren SM. Morphological 3-dimensional assessment, pre-operative simulation and rationale of intra-operative navigation on orthopaedic surgery: Practical application for reorientating osteotomies of the hip joint. Injury 1997; 28, Suppl. 2: $12-30$

${ }^{16}$ Langlotz F, Bacheler R, Berlemann U, Nolte LP, Ganz R. Computer assistance for pelvic osteotomies. Clin Orthop 1998; 354: $92-$ 102

${ }^{17}$ Lehmann T, Oberschelp W, Pelikan E, Repges R. Bildverarbeitung für die Medizin. Springer, Berlin Heidelberg 1997

${ }^{18}$ Liener UC, Reinhart C, Kinzl L, Gebhard F. A new approach to computer guidance in orthopaedic surgery using real time volume rendering. J Med Syst 1999; 23: 35-40

${ }^{19}$ Matsushita S, Suzuki N. Application of computer assisted 3D imaging simulation for surgery. Nippon Rinsho 1994; 52: 817 -823

${ }^{20}$ McGurk M, Amis AA, Potamianos P, Goodger NM. Rapid prototyping techniques for anatomical modelling in medicine. Am J Coll Surg Engl 1997; 79: 169-174

${ }^{21}$ Mittelmeier W, Peters P, Ascherl R, Gradinger R. Rapid Prototyping. Modellherstellung zur präoperativen Planung von rekonstruktiven Beckeneingriffen. Orthopäde 1997; 26: 273-279

${ }^{22}$ Nolte LP, Langlotz F. Intraoperative Navigationssysteme. Trauma Berufskrankh 1999; 1 : $108-115$

${ }^{23}$ Oyama H, Wakao F, Takahira Y. The clinical advantages of editable real-time volume rendering in a medical virtual enviroment:
VolMed. Stud Health Technol Inform 1998; 50: $341-345$

${ }^{24}$ Paley D, Herzenberg JE, Tetsworth K, McKie J, Bhave A. Deformity planning for frontal and sagittal plane corrective osteotomies. Orthop Clin North Am 1994; 25: 425-465

${ }^{25}$ Potamianos P, Amis PP, Forester AJ, Mc Gurk $\mathrm{M}$, Bircher M. Rapid prototyping for orthopaedic surgery. Proc Inst Mech Eng 1998; 212: $383-393$

${ }^{26}$ Reinhart C, Günther T, Poliwoda C, Hesser J Männer R. Volumendatenvisualisierung in Echtzeit. In: Kinzl L, Rehm KE (Hrsg.): Hefte zu Der Unfallchirurg 272, Springer, Berlin Heidelberg New York 1998: S. 541 - 543

${ }^{27}$ Saad A, Hevler A. Das plastische Panoptikum. c't 1999; 7: 164-171

${ }^{28}$ Sailer HF, Haers PE, Zollikofer CP, Warnke T, Carls FR, Stucki P. The value of stereolithographic models for preoperative deformities and planning of surgical corrections. Int J Oral Maxillofac Surg 1998; 27: 327-333

${ }^{29}$ Udupa JK, Hung HM, Chuang KS. Surface and volume rendering in three-dimensional imaging: a comparison. J Digit Imaging 1991; 4: 159-168

${ }^{30}$ Winder J, Cooke RS, Gray J, Fannin T, Fegan T. Medical rapid prototyping and 3D CT in the manufacture of custom made cranial titanium plates. J Med Eng Technol 1999; 23: 26 28

${ }^{31}$ Xia J, Ip HH, Samman N, Wang D, Kot CS, Yeung RW, Tideman H. Computer-assisted three-dimensional surgical planning and simulation: 3D virtual osteotomy. Int J Oral Maxillofac Surg 2000; 29: 11 - 17

32 Zwipp H, Dahlen C, Gavlik JM. Osteotomien am Fußskelett nach in Achsabweichung verheilten Frakturen. OP-Journal 2000; 16: 72 78

\section{Dr. med. Christian Dahlen}

Ltd. Oberarzt

Dr. med. Michael Amlang Oberarzt

Prof. Dr. med. Hans Zwipp

Klinikdirektor

Klinik für Unfall- und Wieder-

herstellungschirurgie

Universitätsklinikum Carl Gustav Carus der Technischen Universität Dresden

Fetscherstr. 74

D-01307 Dresden 